\title{
Foundation dentists supporting vaccination programmes in England
}

\author{
Stefan T. Serban, ${ }^{* 1}$ Sally Eapen Simon, ${ }^{2}$ Karen Vinall-Collier ${ }^{1}$ and Gail V. A. Douglas ${ }^{1}$
}

\section{Key points}

Key stakeholders planning and delivering the 2020/21 flu vaccination programme in Yorkshire and the Humber recognised a workforce gap created by programme expansion as part of the COVID-19 pandemic response. Foundation dentists were invited to support the flu programme and later, the COVID-19 vaccination programme.
Strong system leadership and collaboration between a wide range of healthcare organisations supported innovative solutions for training, indemnity, hostemployment arrangements and competency signoff. Foundation dentists mobilised for flu vaccinations were ready to be rapidly transferred to support the COVID-19 vaccination programme.

\begin{abstract}
Background In 2020/21, as part of the COVID-19 pandemic response and for the first time in England, newly qualified foundation dentists (FDs) were trained to participate in flu and COVID-19 vaccination programmes to offer additional workforce capacity. The largest of these efforts was in Yorkshire and the Humber where 106 FDs were trained and ready to mobilise. The aim of this service evaluation was to appraise the use of FDs in delivering vaccinations.
\end{abstract}

Methods Mixed methods using an online questionnaire to FDs and in-depth remote interviews conducted with host organisations, Public Health England, Health Education England and others.

Results The questionnaire response rate was $89 \%(n=94)$, with 54 FDs having participated in vaccinations at a rate of 50-100 vaccines per day. All were confident with flu vaccine administration and most $(n=44 / 54)$ with COVID-19 vaccination. Eleven stakeholder interviews were conducted. Main barriers included the siloed delivery of dental care from other health services, resulting in collaborative barriers and a lack of understanding about the profession's skillsets. Facilitators included host organisations' capacity to hold multiple honorary contracts and provide competency sign-off.

Conclusion Utilising the dental workforce to deliver vaccinations was feasible at a time of crisis and when trainees' access to dental patients was limited.

\section{Introduction}

Impact of COVID-19 on the healthcare sector

The COVID-19 pandemic had a devastating effect on society from most perspectives. Between March 2020 and March 2021, more than 120,000 people died within 28 days of a positive COVID-19 test in the UK. ${ }^{1}$ The pandemic created significant pressures on the capacity of the NHS to deliver safe and effective care. ${ }^{2}$ To face this unprecedented

'School of Dentistry, University of Leeds, Clarendon Way, Leeds, LS2 9LU, UK; ${ }^{2}$ Public Health England, North East and Yorkshire Region, Blenheim House, Duncombe Street, Leeds, LS1 4PL, UK.

${ }^{\star}$ Correspondence to: Stefan T. Serban

Email address: s.t.serban@leeds.ac.uk

Refereed Paper.

Accepted 27 August 2021

https://doi.org/10.1038/s41415-021-3758-5 challenge, the NHS demonstrated remarkable creativity and resilience through dedication and sacrifices made by its staff and by the wider society. ${ }^{3}$

\section{Impact of COVID-19 on dentistry}

Dental care services were not immune to the impact of the pandemic. ${ }^{4,5}$ In England, lessons learned from the first wave of the pandemic enabled the continuation of the delivery of dental care services throughout the second wave, supported by guidance published by Public Health England (PHE) and the Office of the Chief Dental Officer. ${ }^{6,7}$ Dental care services demonstrated a remarkable ability to adapt to the new measures such as screening and triage, social distancing, fallow time after aerosol generating procedures etc. However, these new measures, together with workforce pressures caused by staff shortages due to the pandemic, the significant reduction in the number of patients and the availability of surgery space, had a significant impact on the availability of training opportunities for dentists, especially for those in foundation training. ${ }^{8,9}$

\section{Need for action}

Every winter, the NHS is under significant pressures due to seasonal influenza. Additionally, in 2020/21, a significant proportion of resources were directed towards the pandemic response. To reduce the risk of the dual threat caused by seasonal influenza and COVID-19, the UK government expanded the eligibility criteria for flu vaccinations to include additional cohorts, for example those over the age of 50, shielding households and all school year groups up to Year 7. Furthermore, the targets for uptake in each cohort were set to minimum $75 \%$, making the 2020/21 flu programme the largest one to date in the history of the NHS. ${ }^{10}$ Based on NHS England estimates for the North East 
and Yorkshire, the new eligible cohorts and the increased ambitions for uptake meant that more than two million extra people became eligible. ${ }^{11}$ The administration of a significant number of additional flu vaccinations in a COVID-safe environment presented challenges.

Dentists are highly-trained professionals and are considered ideally placed to support vaccination programmes. ${ }^{12,13,14}$ Therefore, as part of the pandemic response, in Yorkshire and the Humber (Y\&H) a working group was formed to explore the use of foundation dentists (FDs) to help deliver vaccinations. After obtaining information about appropriate governance for the FDs' involvement, oneday training was delivered to all new $\mathrm{Y} \& \mathrm{H}$ FDs online by Leeds Beckett University and was funded by Y\&H Integrated Care Systems (ICSs). After training, all FDs were invited to participate in vaccination programmes and a practical competency sign-off was provided by each host organisation before allowing FDs to take part. This paper reports the service evaluation conducted, which includes multiple stakeholder points of view on the experiences of the training and deployment of FDs.

\section{Aims and objectives}

The aim was to appraise the use of FDs in delivering vaccinations in $\mathrm{Y} \& \mathrm{H}$. The following objectives were developed:

- Report on FD experiences and their contribution to vaccination programmes

- Seek in-depth views from key stakeholders involved in the development and implementation of the programme.

\section{Methods}

This service evaluation was conducted between October 2020 and April 2021 and was led by a specialist registrar (STS) supervised by an academic consultant in dental public health from the University of Leeds (GVAD) with the support of the Y\&H Academic Health Sciences Network. Using the Health Research Authority decision tool, this project was not classified as original research, therefore ethical review was not required. ${ }^{15}$

An online survey with multiple-choice and free-text questions was designed to record the number of FDs who took part in vaccination programmes and to explore their experiences. After piloting and refinement, this was emailed to all FDs (Figures 1 and 2).

Descriptive statistics were used to analyse quantitative data and a manifest qualitative content analysis was performed on the freetext questions according to recommendations of Krippendorff. ${ }^{16}$

Purposive sampling was used to recruit interviewees from various stakeholder organisations including PHE and Health Education England, FD educational supervisors and host organisations, including those who declined to take part in the programme. One-to-one semi-structured interviews explored their views by telephone or Microsoft Teams. With consent, they were audio-recorded, transcribed verbatim and

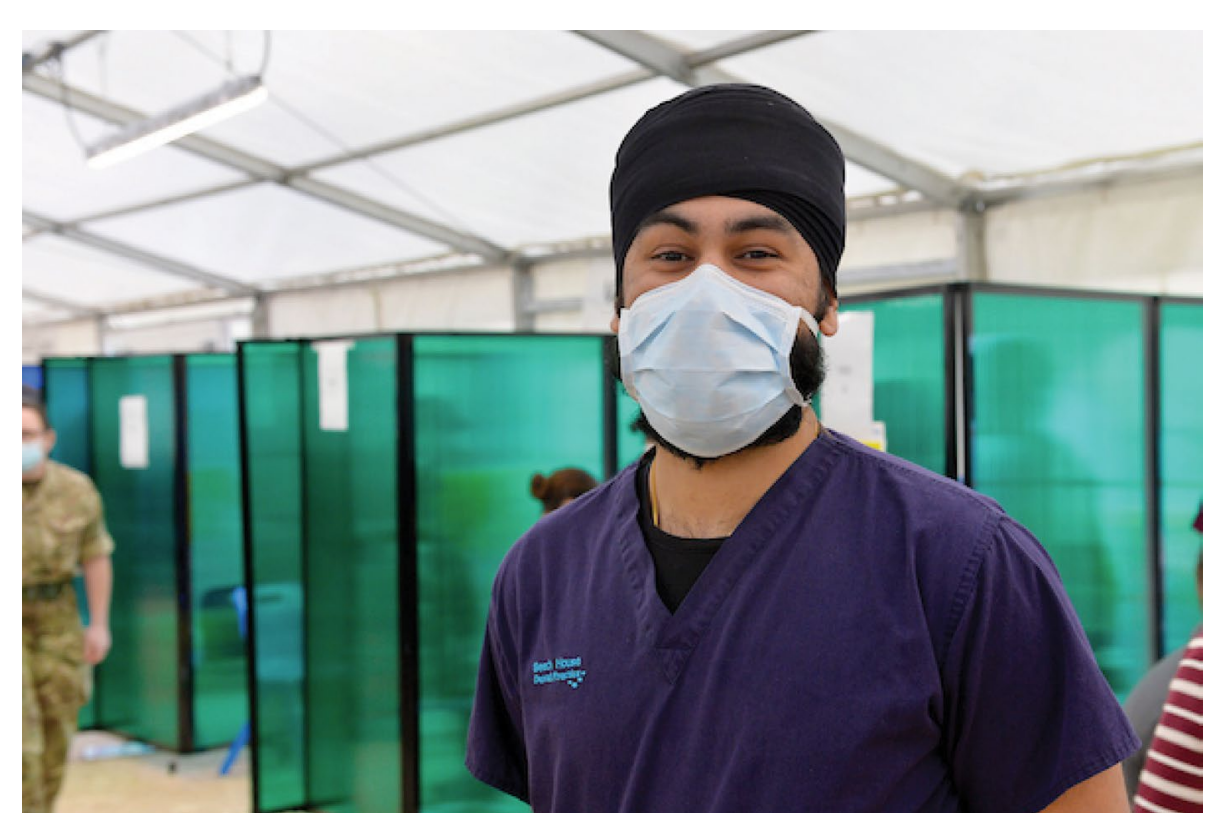

Fig. 1 One of the foundation dentists delivering vaccinations. Image courtesy of Darren Casey

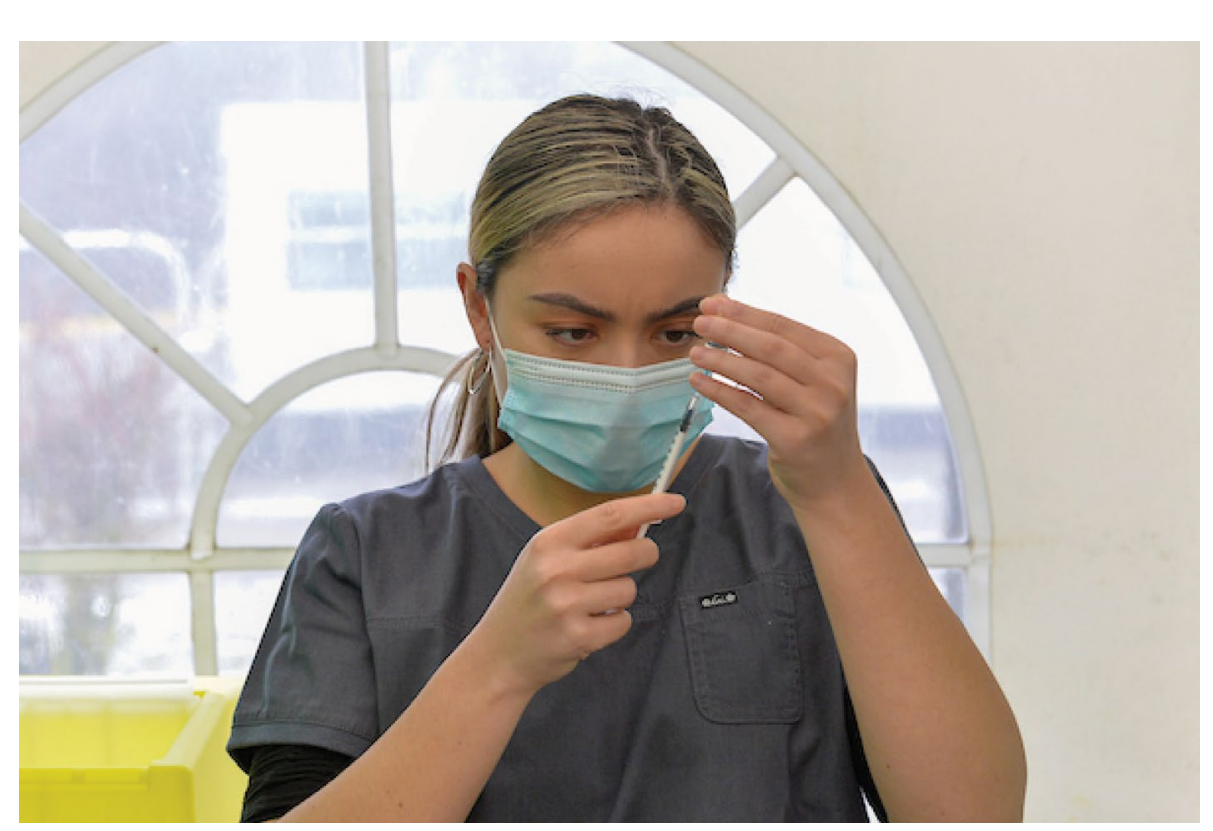

Fig. 2 One of the foundation dentists delivering vaccinations. Image courtesy of Darren Casey 
and included training for all immunisation programmes, not only for flu. ${ }^{17}$

Host organisations were offered FDs for one day per week from early October 2020 until the end of March 2021. The vaccination settings included general practice (GP) surgeries and mass vaccination sites. The pace of mobilisation varied depending on the host organisations' capacity to provide host-employment arrangements and competency sign-off.

\section{Foundation dentists' experiences}

The FD questionnaire had a response rate of $89 \%(n=94 / 106)$. Around half $(n=54)$ of all FDs trained reported being used in delivering vaccinations. In December 2020, when COVID-19 vaccination began, 31 FDs supported both flu and COVID-19 vaccination delivery and 16 were administering only COVID-19 vaccinations.

All 54 FDs who delivered vaccinations reported being confident administering flu vaccinations and 44 of them were confident administering COVID-19 vaccinations. Some FDs described specific technical issues with the COVID-19 vaccinations which were shared by other vaccinator staff: 'the Pfizer vaccination preparation requires the mixture of powder with liquid, following by drawing solution into ten equal doses of injections. I was least comfortable with this aspect'.

The different systems used by providers to record numbers of vaccinations made it challenging to keep an accurate record of the numbers of vaccinations delivered by FDs. Feedback from host organisations and FDs suggests that most of them were delivering around 50 vaccinations per day, with some FDs at mass vaccination sites delivering over 100 per day.

Regarding the impact of the programme on their skills, 51 reported improvements in their communication skills (both with patients and with colleagues) and their teamworking skills through this placement. Thirty-eight FDs reported that the skills gained through this placement were transferable to dentistry. Most FDs $(n=50)$ reported being confident advocating vaccinations to their friends and family.

Some of the main themes emerging about ways to improve the placement were around host organisations understanding the role and skillset of FDs, for example 'we need to find a way of encouraging local GPs/service providers to use us as an excellent resource to administer vaccines, as I feel like the placements did not know enough about us... and didn't have much faith in our abilities to administer an intramuscular injection despite thorough training. Finding a way to get the Clinical Commissioning Group (CCG) to trust what we can do and also use us in a more effective way will be useful for the next flu season or for other vaccine programmes in the future'.

\section{Challenges to mobilisation}

Of the 106 FDs trained to deliver vaccinations, 40 were not utilised by the host organisations. All 40 FDs completed the survey and provided feedback about the reasons for not being mobilised for vaccinations. The main themes emerging from their feedback were around lack of understanding from providers about the role of FDs:

- 'I attended one session with the flu vaccine team. They told me I wasn't allowed to provide any vaccinations. I also signed up to be part of the COVID-19 vaccine team but am still awaiting correspondence from them'.

Other FDs described misunderstandings around flexibility of the offer:

- 'I did ask a couple of times but was not required. On some days I was asked on short notice and had a full day of patients booked.

\section{Successful mobilisation}

Mobilisation across all regions was facilitated by having one lead organisation to host the trainees, though the organisations varied by region. The key aspect of success common to all areas was organisational capacity to resolve host-employment arrangements and competency sign-off for the allocated FDs. The first area to use FDs was Humber Coast and Vale with mobilisation from mid-October and competency sign-off for all 29 allocated trainees. One GP federation there offered to hold the honorary contracts for all trainees in that region and they set-up memoranda of understanding with the other CCGs to allow FDs mobilisation in their area. Ultimately, the other CCGs failed to mobilise the offer and all FDs were utilised by the federation. In this way, the GP federation was pivotal to successful mobilisation with FDs in the vaccination programme within that area.

\section{Stakeholder feedback}

Eleven participants from various organisations consented to be interviewed as part of this evaluation (Table 1).

The interviews were conducted by a speciality registrar in dental public health around the following main topics:
- Value for FDs and for other stakeholders

- Barriers and facilitators for engaging with this project.

\section{Value for foundation dentists}

Participants talked about the opportunity to work in a different setting as an enhanced training experience for FDs, particularly as this provided them with a wider population health experience and a better understanding of the organisational arrangements of the wider primary care system and forged collaborative relationships with other healthcare professionals:

- 'Up until December, most [dental practices] were struggling to give them three days a week let alone five days a week so we felt having a day linked to public health would be a good utilisation of their time...broadening our students' horizons about what it means to be a dentist but also helping the public understand... what dentists can do and how we can support the NHS'

A few participants expressed concerns about the clinical experience of new graduates and the further limitations caused by the pandemic:

- 'Clinical exposure of newly qualified dental graduates is not what it was 20 years ago. So, I think every minute spent in a dental environment to me is more beneficial.

\section{Value for organisation}

General practices that hosted FDs reported that this extra-workforce capacity allowed the existing primary care staff to focus on primary care work and welcomed the flexibility of FDs to do overtime to help speed up the pace of the immunisation programme:

- 'The nurses and the doctors could carry on with their normal appointments on those days...they were able to focus on the bloods

Table 1 Participants' semi-structured interviews

\begin{tabular}{l|l}
\hline Organisation & Participants \\
\hline Public Health England & 2 \\
\hline Health Education England & 2 \\
\hline Educational supervisors & 2 \\
\hline GP practice & 1 \\
\hline Participating CCG & 2 \\
\hline Non-participating CCG & 2 \\
\hline Total & $\mathbf{1 1}$ \\
\hline
\end{tabular}


and the whatever else they were doing. The doctors could focus on the illness and the long-term conditions instead'.

In addition, participants reported that there was some learning in recognising the value of bringing colleagues outside primary medical care into their team.

- 'If we start with the individuals... they've been brilliant actually...it's a completely diverse team working together for one single objective and I've never seen that before in my twenty years as a general practitioner...I think there's something there that we need to capture and not let go actually'.

\section{Barriers and facilitators for engagement with the programme}

The main barriers highlighted by participants were around motivating people to engage and resistance to change. This was reported by eight participants. Another barrier was represented by the complexities around hostemployment arrangements, competency sign-off and the lack of understanding of the FDs training and skillset. This view was shared by eight participants and it was consistent with feedback from the FD survey. Additionally, host organisations had varying capacity for setting-up multiple honorary contracts, facilitating competency sign-off and had varying vaccine supply issues. Five participants highlighted communication issues between host organisations and FDs, as well as between host organisations, the project team and other stakeholders. Also, five participants reported that structural differences between providers and the different delivery models were a potential barrier. The absence of an existing framework and the high-paced implementation were considered barriers by four participants. Other barriers were the lack of an existing precedent, project complexity, lack of flexibility around vaccination days, interprofessional barriers, lack of resources and organisational politics.

The main facilitator reported by five participants was the project team. Two participants discussed the support of various organisations such as Health Education England, NHS England and Improvement, Senior Responsible Office leads and ICSs, local medical committees, educational supervisors etc. Having large healthcare federations be able to host the honorary contracts and large vaccination sites, as well as FDs' professionalism, communications skills and ability to integrate in multidisciplinary teams, were also considered a facilitator by two participants. Other facilitators were the sense of contribution to the pandemic response and the low risk of delivering the programme by understanding the skills and training of FDs.

\section{Discussion}

To our knowledge, this was the first time that foundation dentists were used to support the delivery of the flu programme in England. This highly complex initiative applied the principles of system leadership, involving several different organisations working together towards a shared goal and providing innovative solutions to a complex problem. ${ }^{18}$ This was important as governance arrangements were initially unclear and difficult to ascertain. Confirmation of crown indemnity under the Clinical Negligence Scheme for General Practice was eventually obtained from NHS Resolution.

An important advantage was the strong dental public health leadership to drive the efforts to maximise the offer and the support of the newly formed ICS Flu Boards. This was further helped by the lead consultant having a dual role as both consultant in dental public health and screenings and immunisations lead (SES). This was a key advantage for facilitating the communication between the various organisations involved in the vaccination programmes and the dental stakeholders. A steering group provided the tools for organisations to develop local arrangements and share best practice. It was important to identify and engage with early adaptors and innovators to champion against organisational resistance to change and promote working across the professional boundaries created by the healthcare system. ${ }^{19}$

Feedback from receiving organisations and FDs suggested that one of the main barriers was the lack of understanding of the FD skillset. The detached delivery of dental care from other healthcare services contributed to a lack of understanding of the dental workforce skillset. Future research should explore opportunities for addressing these barriers, especially in the light of the changing demographics and increasing numbers of people with multimorbidities who might benefit from a more person-centred, multidisciplinary approach between healthcare professionals. ${ }^{20,21}$

The main limitation of this evaluation was around the collection of FD activity data. Different immunisation providers used different systems to record the numbers of vaccinations, raising significant challenges for data collection. FD activity regarding vaccinations was based on estimates provided by host organisations.

Although the overall response rate to the FD questionnaire was high, fewer FDs from North Yorkshire responded $(n=17 / 29)$. This might have skewed some of the results, as local intelligence suggests that North Yorkshire had the highest mobilisation of FDs from the three regions in $\mathrm{Y} \& \mathrm{H}$.

Dentists and dental care professionals have a professional responsibility to provide preventative interventions around diet, smoking and alcohol in order to reduce the burden of the associated conditions on the wider healthcare system. ${ }^{22,23}$ Our findings suggest there could be scope to further explore contractual arrangements to include vaccinations as part of dental appointments, for example under flexible (transformational) commissioning arrangements. ${ }^{24}$ These could be delivered cost-effectively by utilising other members of the dental team, for example trained, competent and indemnified nurses or hygienists, in the same manner as in general practices where vaccinations are often provided by nurses.

Some of the criticism around the FD vaccination programme was that 'dentists should be doing dentistry'. Dental care provision in the context of the new Standard Operating Procedures and Infection Prevention and Control Guidance resulted in a significant reduction of the number of patients that could be seen by FDs. ${ }^{6,7}$ Anecdotal evidence suggests that certain FD training practices could only offer training opportunities on a limited number of days a week in autumn 2020. The resulting workforce capacity created by this unique situation was utilised in a creative manner to identify opportunities to fulfil FD training competencies. By mobilising the workforce to do 'the right thing at the right time' and support the pandemic response in a 'Dunkirk spirit', FDs had a unique opportunity to be involved in the wider structures of primary care and demonstrate the value of collaboration between medicine and dentistry. ${ }^{25}$

The pandemic provided a circuit breaker in our daily routine and an opportunity to reflect on our role and the value of our practice. ${ }^{26}$ Structural, cultural and economic change that goes beyond dental contract reform is needed with a strong focus on prevention and reduction of inequalities. ${ }^{21,24}$ 
The upcoming imminent changes in the NHS present an opportunity for commissioning organisations and regulatory bodies to consider new structures for a more integrated care where dentistry is an active part of the primary care offer in order to support a person focused approach. ${ }^{27,28,29}$ As Davie Hollis said, in the rush to return to normal, use this time to consider which parts of normal are worth rushing back to. ${ }^{30}$

\section{Conclusion}

The results of the evaluation suggest that the model for utilising dental workforce in primary medical care settings to deliver vaccinations is feasible. One of the challenges which remains is the siloed delivery of dental care from other health services resulting in collaborative barriers and a lack of understanding about the professions skillsets. Further exploration is necessary to assess whether delivery of vaccinations within the dental care setting is feasible.

\section{Ethics declaration}

The authors have no conflicts of interests.

\section{Acknowledgements}

We wish to express our gratitude to Richard Stubbs and his team, especially Helen Hoyland and Neil Toner from the Yorkshire and the Humber Academic Health Sciences Network for their generous support for this service evaluation. Special thanks to James Spencer and Jason Atkinson from Health Education England Yorkshire and the Humber, Dr Siobhan Grant from Public Health England North East and Yorkshire, Professor Mike Holmes and his team from Nimbuscare Ltd and Alex Morton and Emma Wilson from NHS England and NHS Improvement Yorkshire and the Humber. Also, we would like to acknowledge the support provided by Dr Olivia Barraclough, Dr Zhain Mustufvi, Ms Sarah Simon and Dr Joshua Twigg, the educational supervisors and training programme directors and the 106 foundation dentists and the numerous other organisations supporting this complex project.
Author contributions

Stefan T. Serban led the evaluation, collected the data, wrote the first draft of the paper and agreed the final version. Sally E. Simon led the project, contributed to the design of evaluation, contributed to the drafts and agreed the final version. Karen Vinall-Collier contributed to the design of the evaluation, reviewed drafts and agreed the final version. Gail V. A. Douglas advised on the design of the evaluation, reviewed drafts and agreed the final version.

\section{References}

1. UK Government. Deaths within 28 days of positive test. 2021. Available at https://coronavirus.data.gov.uk/ details/deaths (accessed March 2021).

2. McCabe R, Schmit N, Christen P et al. Adapting hospital capacity to meet changing demands during the COVID19 pandemic. BMC Med 2020; 18: 329.

3. British Medical Association. Pressure points in the NHS. 2021. Available at https://www.bma.org.uk/ advice-and-support/nhs-delivery-and-workforce/ pressures/pressure-points-in-the-nhs (accessed March 2021).

4. Coulthard P, Thomson P, Dave M, Coulthard F P, Seoudi $N$, Hill M. The COVID-19 pandemic and dentistry: the clinical, legal and economic consequences - part 2: consequences of withholding dental care. Br Dent J 2020; 229: 801-805.

5. Coulthard P, Thomson P, Dave M, Coulthard F P, Seoudi $\mathrm{N}$, Hill M. The COVID-19 pandemic and dentistry: the clinical, legal and economic consequences - part 1: clinical. Br Dent J 2020; 229: 743-747.

6. UK Government. COVID-19: infection prevention and control dental appendix. 2020. Available at https:// www.gov.uk/government/publications/wuhan-novelcoronavirus-infection-prevention-and-control/covid19-infection-prevention-and-control-dental-appendix (accessed March 2021)

7. Office of the Chief Dental Officer England. Standard operating procedure Transition to recovery. 2021. Available at https://www.england.nhs.uk/coronavirus/ wp-content/uploads/sites/52/2020/06/C1461-dentaltransition-to-recovery-sop-v6.pdf (accessed March 2021).

8. British Dental Association. Coronavirus: Foundation dentists and the impact of the COVID-19 crisis. 2020 Available at https://bda.org/news-centre/blog/ coronavirus-foundation-dentists-and-the-impact-ofthe-covid-19-crisis (accessed March 2021).

9. Mahendran K, Yogarajah S, Herbert C, Nayee S, Ormond M. COVID-19 and Postgraduate Dental Training - A commentary. Eur J Dent Educ 2020; 25: 415-419.

10. Department of Health and Social Care. Most comprehensive flu programme in UK history will be rolled out this winter. 2020. Available at https://www. gov.uk/government/news/most-comprehensive-fluprogramme-in-uk-history-will-be-rolled-out-this-winter (accessed March 2021)

11. Office for National Statistics. Population estimates. 2021. Available at https://www. ons.gov.uk/peoplepopulationandcommunity/ populationandmigration/populationestimates (accessed April 2021).
12. Serban S, Eapen-Simon S, Grant S. Dental team immunisation. Br Dent J 2020; 229: 209.

13. Serban S, Mustufvi Z, Kang J, Simon S E, Grant S, Douglas G. The Dental Team: An Additional Resource for Delivering Vaccinations. Front Med (Lausanne) 2020; 7: 801.

14. Sacoor S, Chana S, Fortune F. The dental team as part of the medical workforce during national and global crises. BrDent J 2020; 229: 89-92.

15. Medical Research Council and NHS Health Research Authority. Is my study research? 2020. Available at http://www.hra-decisiontools.org.uk/research/ (accessed March 2021).

16. Krippendorff K. Reliability in Content Analysis: Some Common Misconceptions and Recommendations. Hum Commun Res 2004; 30: 411-433.

17. Public Health England and Royal College of Nursing. National Minimum Standards and Core Curriculum for Immunisation Training for Registered Healthcare Practitioners. 2018. Available at https://assets. publishing.service.gov.uk/government/uploads/ system/uploads/attachment_data/file/679824/ Training_standards_and_core_curriculum_ immunisation.pdf (accessed March 2021).

18. Edmonstone J. Systems leadership in health and social care. Oxfordshire: Routledge, 2019.

19. Rogers E M. Diffusion of innovations. New York: Simon and Schuster, 2010.

20. Starfield B. Is patient-centred care the same as personfocused care? Perm J 2011; 15: 63-69.

21. Watt R G, Serban S. Multimorbidity: a challenge and opportunity for the dental profession. Br Dent J 2020; 229: 282-286.

22. Public Health England. Delivering better oral health: an evidence-based toolkit for prevention, 3rd ed. 2021. Available at https://www.gov.uk/government/ publications/delivering-better-oral-health-anevidence-based-toolkit-for-prevention (accessed April 2021).

23. National Institute for Health and Care Excellence. Oral health promotion: general dental practice. 2015 Available at https://www.nice.org.uk/guidance/ng30 (accessed April 2021).

24. Mustufvi Z, Barraclough O, Hearnshaw S et al. Flexible Commissioning: A prevention and access focused approach in Yorkshire and the Humber. BDJ In Pract 2020; 33: 20-22.

25. Kay E. One year on. Evid Based Dent 2021; 22: 4.

26. Hurley $S$. Why re-invent the wheel if you've run out of road? Br Dent J 2020; 228: 755-756.

27. Department of Health and Social Care. Integration and innovation: working together to improve health and social care for all. 2021. Available at https:// www.gov.uk/government/publications/workingtogether-to-improve-health-and-social-care-for-all/ integration-and-innovation-working-together-toimprove-health-and-social-care-for-all-html-version (accessed April 2021).

28. Willcocks S, Willcocks E. The shift to integrated care in the NHS: implications of the new care models for dentistry. Br Dent J 2019; 226: 319-322.

29. British Dental Journal. Enhanced role for oral health in imminent NHS long-term plan. Br Dent J 2018; 225: 689.

30. Hollis $D$. In the rush to return to normal, use this time to consider which parts of normal are worth rushing back to. 2020. Available at https://twitter.com/ MrDaveHollis/status $/ 1245361542985637894 ? s=20$ (accessed April 2021). 